\title{
Immune monitoring after pediatric liver transplantation - the prospective ChilSFree cohort study
}

\author{
Imeke Goldschmidt ${ }^{1}$, André Karch ${ }^{2 *}$ D, Rafael Mikolajczyk ${ }^{2,10}$, Frauke Mutschler ${ }^{1}$, Norman Junge ${ }^{1}$, \\ Eva Doreen Pfister ${ }^{1}$, Tamara Möhring ${ }^{1,2}$, Lorenzo d'Antiga ${ }^{3}$, Patrick McKiernan ${ }^{4,11}$, Deirdre Kelly ${ }^{4}$, \\ Dominique Debray ${ }^{5}$, Valérie McLin ${ }^{6}$, Joanna Pawlowska ${ }^{7}$, Loreto Hierro ${ }^{8}$, Kerstin Daemen ${ }^{9}$, Jana Keil ${ }^{9}$, \\ Christine Falk ${ }^{9}$ and Ulrich Baumann ${ }^{1,4}$
}

\begin{abstract}
Background: Although trough levels of immunosuppressive drugs are largely used to monitor immunosuppressive therapy after solid organ transplantation, there is still no established tool that allows for a validated assessment of functional degree of immunosuppression or the identification of clinically relevant over- or under-

immunosuppression, depending on graft homeostasis. Reliable non-invasive markers to predict biopsy proven acute rejection (BPAR) do not exist. Literature data suggest that longitudinal measurements of immune markers might be predictive of BPAR, but data in children are scarce. We therefore propose an observational prospective cohort study focusing on immune monitoring in children after liver transplantation. We aim to describe immune function in a cohort of children before and during the first year after liver transplantation and plan to investigate how the immune function profile is associated with clinical and laboratory findings.

Methods: In an international multicenter prospective approach, children with end-stage liver disease who undergo liver transplantation are enrolled to the study and receive extensive immune monitoring before and at 1, 2, 3, 4 weeks and 3, 6, 12 months after transplantation, and whenever a clinically indicated liver biopsy is scheduled. Blood samples are analyzed for immune cell numbers and circulating levels of cytokines, chemokines and factors of angiogenesis reflecting immune cell activation. Statistical analysis will focus on the identification of trajectorial patterns of immune reactivity predictive for systemic non-inflammatory states, infectious complications or BPAR using joint modelling approaches.

Discussion: The ChilSFree study will help to understand the immune response after pLTx in different states of infection or rejection. It may provide insight into response mechanisms eventually facilitating immune tolerance towards the graft. Our analysis may yield an applicable immune panel for non-invasive early detection of acute cellular rejection, with the prospect of individually tailoring immunosuppressive therapy. The international collaborative set-up of this study allows for an appropriate sample size which is otherwise difficult to achieve in the field of pediatric liver transplantation.
\end{abstract}

Keywords: Paediatric liver transplantation, Immune monitoring, Rejection

\footnotetext{
* Correspondence: andre.karch@helmholtz-hzi.de

${ }^{2}$ Epidemiological and Statistical Methods Research Group, Helmholtz Centre

for Infection Research, Inhoffenstr. 7, 38127 Braunschweig, Germany

Full list of author information is available at the end of the article
}

(c) The Author(s). 2018 Open Access This article is distributed under the terms of the Creative Commons Attribution 4.0 International License (http://creativecommons.org/licenses/by/4.0/), which permits unrestricted use, distribution, and reproduction in any medium, provided you give appropriate credit to the original author(s) and the source, provide a link to the Creative Commons license, and indicate if changes were made. The Creative Commons Public Domain Dedication waiver (http://creativecommons.org/publicdomain/zero/1.0/) applies to the data made available in this article, unless otherwise stated. 


\section{Background}

Successful immunosuppression (IS) in pediatric liver transplantation balances the degree of systemic immunosuppression between under- and over-suppression, minimizing the risks of graft rejection, infection and malignancy. The clinical challenge of how to best define, measure and monitor this optimal state has not been answered other than by clinical experience correlating a desired systemic non-inflammatory state to therapeutic drug monitoring [1].

Numerous approaches have been evaluated in order to monitor the effects of calcineurin inhibition and to guide the dosing of IS medication. An ideal monitoring tool would be easy to use in routine clinical care and yield rapid results that enable dose adjustments and a timely control of their effect. It would be cost-effective and would enable clinicians to navigate the fine individual line between just enough IS to prevent rejection, and as little IS as possible to minimize side effects. Current clinical practice predominantly uses pharmacokinetics, i. e. trough levels of immunosuppressive drugs, sometimes enhanced by $\mathrm{C} 2$ ( $2 \mathrm{~h}$ post dosing) levels and area under the curve (AUC) pharmacokinetics [2]. However, it is recognized that pharmacokinetic drug monitoring does not necessarily reflect the functional degree of immunosuppression. In studies that investigated IL- 2 and IFN- $\gamma$ production in T-cells as well as soluble IFN- $\gamma$, IL- 2 and IL-17 concentrations as measures for the functional degree of immunosuppression, the degree of inhibition of cytokine production was not strongly associated with plasma tacrolimus or mycophenolic acid (MMF) concentrations [3]. Moreover, there was no difference in tacrolimus trough levels and tacrolimus AUC values or MMF AUC values between patients who did or did not reject $[3,4]$.

A number of approaches trying to describe the functional changes in the immune system after initiation of IS therapy have been published [3-7]. The main effect of calcineurin inhibitors is the inhibition of T cell activation by suppressing translocation of the NFAT transcription factor into the nucleus which blocks transcription and secretion of IL-2, GM-CSF and other cytokines from T cells. Therefore, the majority of experimental approaches focus on $\mathrm{T}$ cell activation, proliferation and cytokine secretion. Reduced $\mathrm{T}$ cell activation has been demonstrated in comparisons of adult kidney, heart and liver transplant recipients with either healthy adults [5] or intra-individually with pre and post dosing studies in transplanted patients $2 \mathrm{~h}$ after Cyclosporin A (CSA) ingestion compared to baseline [6, 7].

The measurement of circulating cytokine levels in the peripheral blood plasma represents an indirect measure of $\mathrm{T}$ cell activation, but allows assessing both TH1, TH2 and TH17 patterns at the same time. It also permits to analyze the complex interplay and cross-activation of different immune cells. Studies that measured circulating levels of IFN- $\gamma$, TNF- $\alpha$, IL-2, soluble IL-2 receptor (sCD25), IL-10, IL-4, IL5, IL-12-p70, IL-12p40 and IL-15 at various time points before and after transplantation demonstrated an increase of both TH1 and TH2 cytokines shortly after transplantation, with differences in cytokine levels thereafter reflecting the occurrence of biopsy-proven acute cellular rejection (BPAR) [8-11].

Several other studies have examined immune markers in a longitudinal fashion in order to investigate predictors of BPAR. Observation periods ranged from very short-term (before transplantion (Tx) until 1 week after $\mathrm{Tx})$ to long-term (before Tx until 24 months after Tx). While the results with regards to an association of pretransplant variables with the occurrence of early BPAR are variable $[3,4,12,13]$, most studies reported a strong association of early changes after transplantation with the occurrence of BPAR [3, 11, 12]. In a study that followed liver transplanted adults for up to 1 year after transplantation, IFN- $\gamma$ and IL-2 intracellular staining in T-cells and soluble concentrations compared to baseline ("percentage of inhibition") during first week posttransplant differed between rejectors and non-rejectors [3]. In another group of 47 adult liver transplant recipients, serum concentrations of IFN- $\gamma$ and IL-12-p70/IL12p40 (TH1 cytokines), IL-4, IL-6 and IL-10 (TH2 cytokines) and TGF- 3 (TH3 cytokines) were analyzed pretransplant, $12 \mathrm{~h}$ post-transplant, daily until day 15 and every 3 days from day 15 to 30 . Both rejectors and nonrejectors showed an increase of cytokines early after transplantation (day 3-9); however, TH2 cytokines clearly dominated in non-rejectors whereas levels of TH1 and TH2 were similar with slight dominance of TH1 cytokines in rejectors [11].

The number of studies examining functional immune changes after solid organ transplantation in children is still limited. A significant increase of donor-specific Granzyme B ELISPOT frequencies at day seven posttransplant compared to baseline was linked to the occurrence of BPAR in 28 pediatric liver transplant recipients [12]. Gras et al. [8] performed a prospective analysis in 40 children after LTx, 8 of whom experienced early BPAR. Circulating cytokine levels at 1 and $2 \mathrm{~h}$ after reperfusion and on day $1,4,7,14$ and 28 after Tx were determined. IL- 4 was found to be decreased compared to baseline at all time points in the acceptance group [8].

In a large study incorporating 105 liver transplanted children for cross-sectional analysis with a subgroup of 22 children for longitudinal analysis, circulating cytokine levels at 12 months post-transplant differed between rejectors and non-rejectors. In the longitudinal analysis, there was a marked change in soluble cytokine levels compared to baseline at 4 weeks after transplantation, 
but none thereafter [9]. In addition, there was a strong association of TH11 and TH12 cytokine levels with age that was independent of the occurrence of BPAR.

\section{Aims and objectives}

A clear-cut biomarker panel that provides information on the functional degree of immunosuppression and that may allow individualized prediction of tolerance or the risks of rejection or infection has not been established. In pediatric medicine the respective roles of the innate and the adaptive immune system during growth and development and the background of heterogeneous inherited liver diseases have also not been addressed yet, and the privileged role of the liver in transplantation has not been fully understood. Previous studies suffer from small sample sizes, especially in the area of pediatric solid organ transplantation. The few longitudinal studies published suggest that it is the interplay of immune markers over time rather than an absolute value at any given time point that is associated with the occurrence of tolerance.

It is for these reasons that in 2012 a consortium of seven European pediatric liver transplant centers joined an enprEMA recognized network to create the observational ChilSFree study. ChilSFree monitors immune markers such as cytokine, chemokine and angiogenic protein levels as well as immune cell numbers in peripheral blood from before transplantation until 12 months after transplantation. Our study aims at characterising the features of the innate and adaptive immune system before transplantation and after the introduction of immunosuppressive therapy after liver transplantation over the course of time. It uses a prospective longitudinal approach in order to link changes in the observed immune markers to the occurrence of clinical events such as rejection or infection. The multicenter approach will allow for sufficient numbers of participants. The scientific aim is to define markers of immune functioning that are specific to the functional degree of immunosuppression after liver transplantation which could be tested for their use for clinical management of minimized drug induced immunosuppression in future interventional trials.

\section{Methods \\ Study design}

The study is designed as a prospective, observational cohort study. Children under the age of 18 years who receive de novo liver transplantation at one of seven participating European centers are considered for inclusion in the study; they undergo blood sampling for study purposes before transplantation and at 1,2, 3, and 4 weeks as well as 3, 6 and 12 months after transplantation (Fig. 1). Additional samples are being obtained on the occasion of clinically suspected acute cellular rejection. Blood samples for analysis of immune markers are sent overnight to a central laboratory and are centrally analyzed. Clinical data is recorded locally in a purposebuilt internet and Marvin-based electronic case report form (eCRF).

Immunosuppressive therapy will follow the local protocols and will be adjusted according to clinical needs as per the local protocols. The majority of the participating centres have previously been following very similar immunosuppressive protocols. Five of the participating centres use a standard steroid-free protocol for immunosuppression with Anti IL2R (Simulect; $10 \mathrm{mg}$ intravenously if weight $<35 \mathrm{~kg}, 20 \mathrm{mg}$ if $>35 \mathrm{~kg}$ at postoperative day (POD) 0 and POD4) and Tacrolimus (initially Modigraf, if and when appropriate followed by Prograf; loading dose: $0,1 \mathrm{mg} / \mathrm{kg}$; then $0,05 \mathrm{mg} / \mathrm{kg} / 12 \mathrm{hrly}$; target ranges: week 1 and 2: about 12 (10 to 15$) \mathrm{mg} / \mathrm{l}$, week 3 and 4: about 10 (8 to 12$) \mathrm{mg} / \mathrm{l}$, month 1 to 3: about 8 (6 to 10$) \mathrm{mg} / \mathrm{l}$, month 4 to 12 : about 6 (4 to 8$) \mathrm{mg} / \mathrm{l}$, thereafter about 4 (2 to 6) $\mathrm{mg} / \mathrm{l}$; if cellcept is added, target ranges will be $2 \mathrm{mg} / \mathrm{l}$ lower).

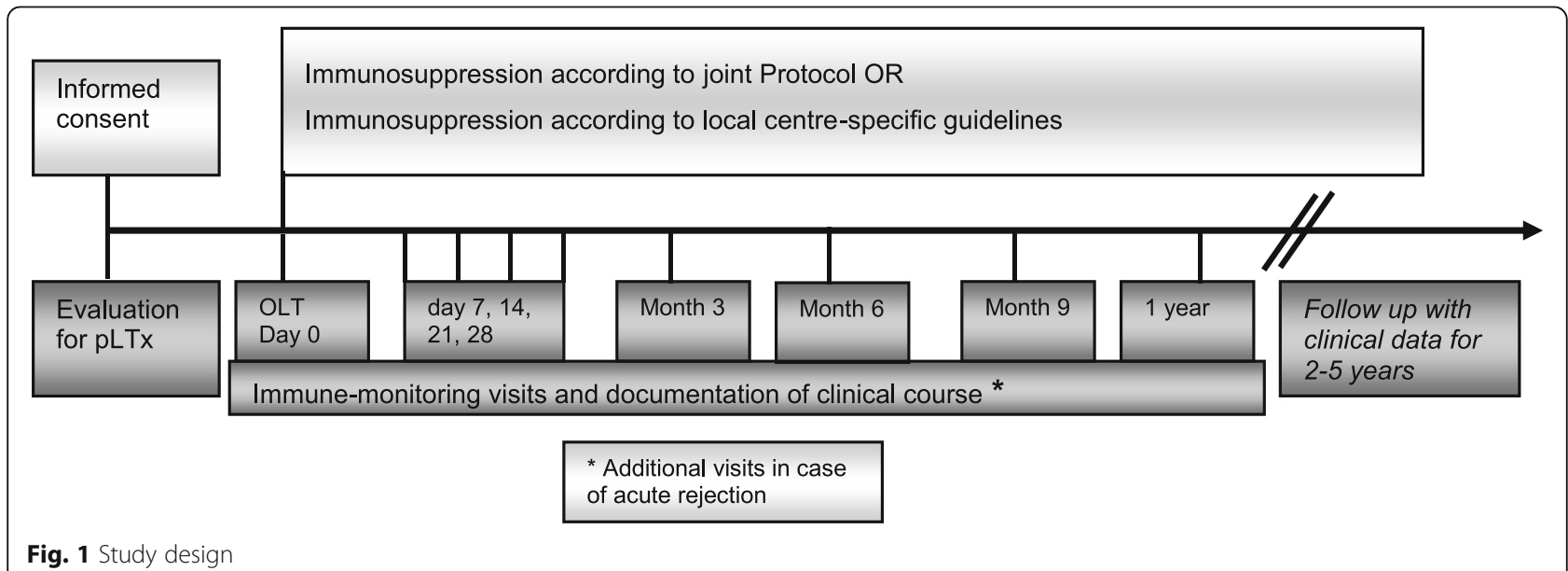

Fig. 1 Study design 
Deviations from the above protocol in all centres will be made as clinically indicated and will be documented in the eCRF. Children will undergo blood sampling for immune monitoring at the occasion of routine clinical visits. Neither additional visits nor additional blood samplings are planned. Medical therapy will be applied as clinically indicated and per the local post-transplantation protocols. No additional interventions are proposed by the observational ChilSFree study protocol.

\section{Study population}

A priori, all patients under the age of 18 years undergoing de novo liver transplantation will be eligible for participation irrespective of primary disease. Exclusion criteria have been defined as underlying conditions that may interfere with the patient's safety, compliance or study evaluation in the opinion of the local investigator and absence of informed consent of parents or legal guardians or the (adolescent) patient. Re-transplantation has also been defined as an exclusion criterion as the study also examines the changes in the immune system compared to the pre-transplant status. In patients who undergo re-transplantation, the pre-transplant-status is not IS naïve.

\section{Immune monitoring}

The study will examine both cellular and humoral immune markers. Subsets of inflammatory cells $(C D 4+$ and CD8+ T cells, CD19+ B cells, CD56/16 + NK cells, CD56 $+\mathrm{T}$ cells, monocytes and granulocytes) will be measured using Trucount analyses with flow cytometry. A set of 50 different serum cytokines, chemokines and factors of angiogenesis reflecting immune cell activation and regulation of immune response will be measured using Luminex-based multiplex assays (Table 1).

\section{Clinical data collection}

Clinical parameters to be documented include demographic data such as age, sex, weight, height and primary disease of the patients, transplant associated information such as the indication for transplantation, donorrecipient relation (living related donation or cadaveric

Table 1 Serum cytokines, chemokines and factors of angiogenesis analyzed in ChilSFree

\begin{tabular}{|c|c|c|c|c|c|}
\hline Cytokines & & Synonym & Chemokines & & Synonym \\
\hline \multirow[t]{6}{*}{ TH1 response } & $\mathrm{IFN}-\gamma$ & & CCL chemokines & CCL2 & MCP-1 \\
\hline & IL-2 & & & CCL3 & MIP1a \\
\hline & $\mid \mathrm{L}-12$ & & & CCL4 & MIP-1b \\
\hline & G-SCF & & & & \\
\hline & GM-CSF & & & CCL5 & RANTES \\
\hline & TNF-a & & & CCL7 & MCP-3 \\
\hline \multirow[t]{4}{*}{$\mathrm{TH} 2$ response } & $\mid \mathrm{L}-4$ & & & CCL11 & Eotaxin \\
\hline & IL-5 & & & CCL27 & CTACK \\
\hline & IL-10 & & CXCL chemokines & CXCL1 & Gro-a \\
\hline & IL13 & & & CXCL8 & $\| \mathrm{L}-8$ \\
\hline TH9 responses & IL-9 & & & CXCL9 & MIG \\
\hline \multirow[t]{2}{*}{ TH17 response } & $\mid \mathrm{L}-17$ & & & CXCL10 & $\mid \mathrm{P}-10$ \\
\hline & IL-23 & & & CXCL12 & SDF-1a \\
\hline \multirow[t]{7}{*}{ polyfunctional } & IL-1a & & Growth factors & M-CSF & \\
\hline & IL-1b & & & SCF & \\
\hline & IL-1RA & & & SCGF & \\
\hline & IL-3 & & & PDGF & \\
\hline & IL-6 & & & HGF & \\
\hline & IL-18 & & & FGF-b & \\
\hline & LIF & & & MIF & \\
\hline \multirow[t]{5}{*}{ Angiogenic factors } & Ang-2 & angiopoietin-2 & Soluble surface molecules & sCD25 & IL-2Ra \\
\hline & VEGF & & & ICAM-1 & \\
\hline & PECAM-1 & sCD31 & & VCAM & \\
\hline & Leptin & & & TRAIL & \\
\hline & & & & Follistatin & \\
\hline
\end{tabular}


transplantation, blood group compatibility), type of graft (split or full-size) and surgical as well as non-surgical complications. Information on infections is collected both as a documentation of the EBV- and CMV serostatus at transplantation, and as documentation of viral and bacterial infections in the course of the study. Immunosuppression will be documented with regards to type of IS medication, dose and trough level at the study visits and changes of IS medication with the respective reasons for changing the therapy. In cases of liver biopsy, presence of acute cellular rejection is noted both as a binary choice (rejection vs no rejection) as well as on an ordinal scale with grading according to the RAI score. A full list of clinical parameters to be documented is given in Table 2 .

Table 2 Clinical parameters recorded in CHilSFree

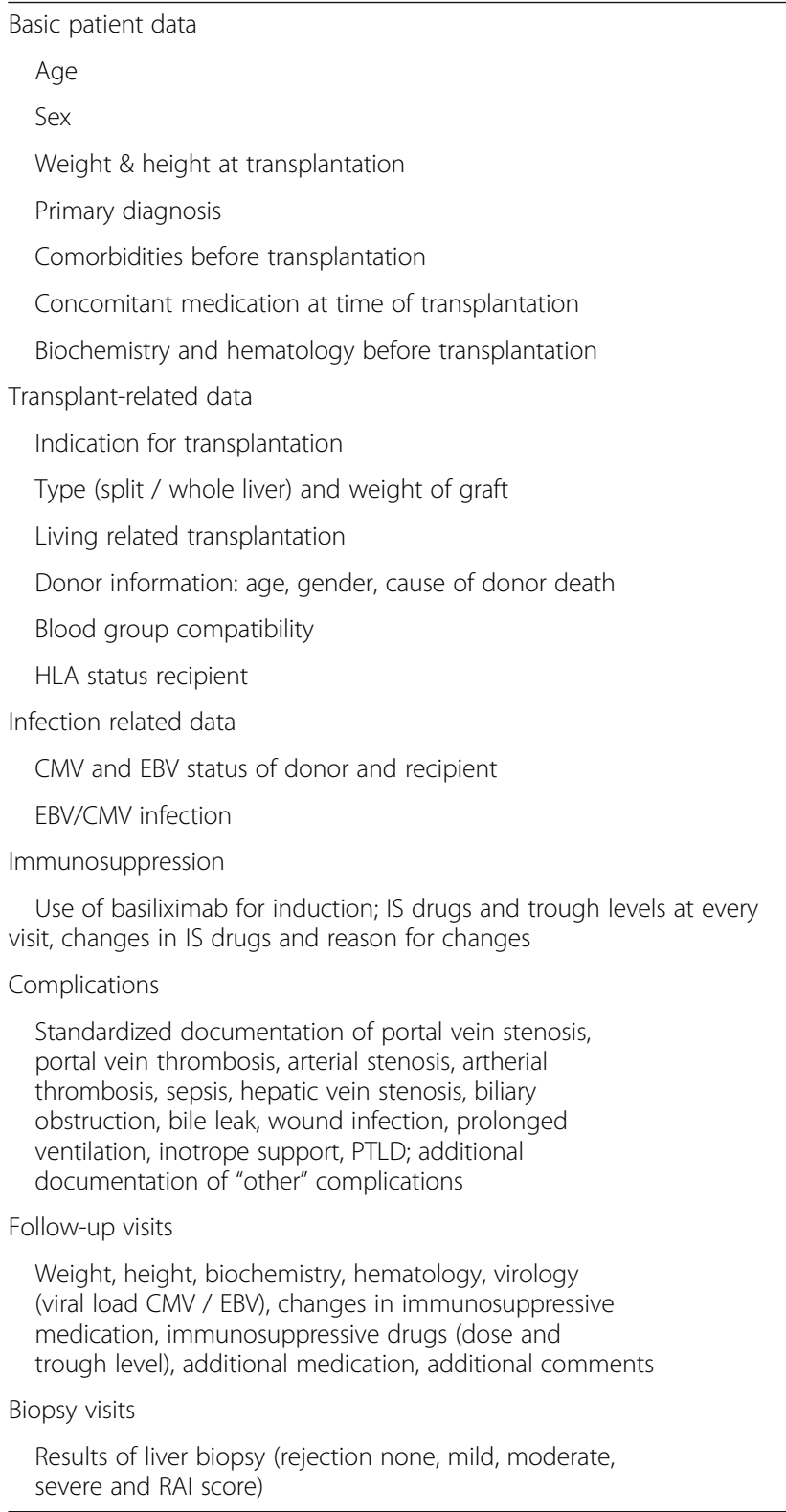

\section{Data management and documentation}

For documentation of clinical parameters, an electronic case report form (eCRF) using the Marvin software (xclinical) was created. Parameters to be documented in the eCRF were compiled using input from all participating centers. After creation and testing of the eCRF, a pilot phase was initiated with data entry from all participating centers, followed by a focus group that discussed feasibility of documentation in everyday clinical practice, unambiguity of data entries and missing items that were deemed to be important. The eCRF was amended accordingly before definitive data capture commenced. Special note in the eCRF amendment was put on the documentation of rejection episodes as this information was regarded as vital for later data analysis. Monitoring of data entries into the eCRF is performed in a stepwise fashion. Participating centers are responsible for correctness of data entry locally and for compliance of their data with the local source data. In addition, a central data monitoring will be provided by the organizing center that will focus on plausibility of data entries and conformity of data documentation between the different participating centers. Queries are sent out centrally to the respective centers as well and will then be answered locally to improve data quality.

\section{Data analysis}

Sample size calculation was originally performed based on the a priori hypothesis that two subgroups with differing risks of rejection (20\% for the low risk group, $60 \%$ for the high risk group, frequency ratio 10:1, overall rejection risk of $25 \%$ ) can be identified using immune monitoring. Taking into account $5 \%$ loss to follow up, 220 participants with available biosamples would be necessary to assess this hypothesis with $90 \%$ power and a two-sided alpha of $5 \%$ (chi squared test) in a confirmatory manner.

However, the main part of the ChilSFree study is designed to be hypothesis generating and focuses therefore on exploratory analyses. This includes the definition of post-transplant trajectories of immune response and the evaluation of the role of these trajectories as predictors for the presence or absence of tolerance. We will use flexible joint modelling approaches [14] in combination with suitable feature selection algorithms for highdimensional data as e.g. boosting [15] or knowledgebased latent classes [16] to build predictive models for time to establish outcomes. Dynamic prediction methods will then be used to assess the predictive potential of the respective models [17].

\section{Description of the study population}

Enrolment for the ChilsFree Study started in December 2012. To date, $n=277$ children have been screened and provided informed consent for the study. Twenty three 
children had to be excluded from the study for various reasons (Fig. 2), leaving 254 study participants (56\% male, $44 \%$ female). Samples for Visit 0 (pre-transplant) are available from 220 patients as requested in the sample size calculation. Participants' age ranged from 0.2 to 18.0 (median 2.5) years. Biliary atresia was the most frequent underlying diagnosis $(n=101,39.8 \%$, Table 3$)$. Living related transplantation was carried out in $n=72$ (28.3\%) cases. The vast majority of transplantations were $\mathrm{ABO}$ compatible, with only $n=13$ being ABO incompatible (5.1\%).

\section{Discussion}

We propose an observational longitudinal study on immune monitoring in children after liver transplantation. Children will be followed from pre transplantation up to 12 months after transplantation and will receive regular extensive immune monitoring assessments. Findings will be linked to clinical changes, especially the occurrence of biopsy proven rejection (BPAR) and infections, with the aim to identify predictors of rejection or infection and delineate candidates for future immune monitoring and guidance of immunosuppressive therapy in clinical practice.

While there is a considerable number of studies that have investigated changes in the immune system after solid organ transplantation, notably with regards to $\mathrm{T}$ cell activation and proliferation, their use for application in children is very limited. Most published studies target adults $[3-7,13,18-22]$ and predominantly investigate
Table 3 Baseline characteristics of the study population

\begin{tabular}{ll}
\hline Subjects & $\mathrm{n}(\%) /$ median (range) \\
\hline Mex & $142(56 \%)$ \\
Female & $112(44 \%)$ \\
Age & 2.5 years $(0.2-18.0)$ \\
Diagnosis & \\
Biliary atresia & $101(39.8 \%)$ \\
Malignant liver disease & $24(9.4 \%)$ \\
Metabolic liver disease & $21(8.3 \%)$ \\
Acute liver failure & $10(3.9 \%)$ \\
PSC & $7(2.8 \%)$ \\
PFIC & $12(4.7 \%)$ \\
Alagille syndrome & $11(4.3 \%)$ \\
Cystic fibrosis & $11(4.3 \%)$ \\
Wilson's disease & $4(1.6 \%)$ \\
Congenital hepatic fibrosis & $3(1.2 \%)$ \\
Toxic liver damage & $1(0.4 \%)$ \\
Other & $49(19.3 \%)$ \\
Type of graft & $73(28.7 \%)$ \\
Living related transplantation & $181(71.3 \%)$ \\
Cadaveric transplantation & \\
ABO compatibility & $241(94.9 \%)$ \\
ABO incompatible & $13(5.1 \%)$ \\
Abbrible &
\end{tabular}

Abbreviations: PSC primary sclerosing cholangitis, PFIC progressive familial intrahepatic cholestasis

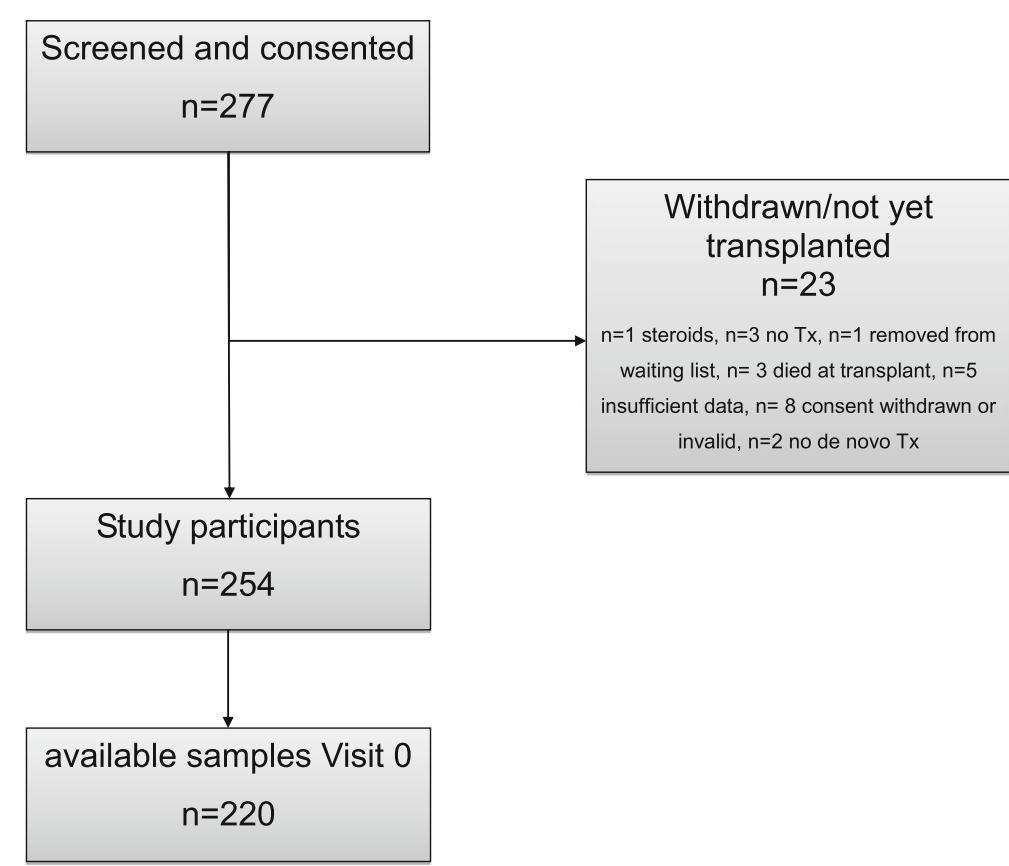

Fig. 2 Flowchart of patient enrolment 
patients after kidney transplantation not liver transplantation $[4,5,13,19,20,23]$. In pediatric liver transplantation a number of differences from adult liver transplantation might affect immune monitoring results warranting a specific pediatric approach. The distribution of primary diseases leading to liver transplantation is different in children where there are hardly any viral hepatitis patients, no alcoholic cirrhosis patients and hardly any NASH/NAFLD patients among the transplant population. Biliary atresia remains the major cause for pediatric liver transplantation ranging from 40 to $80 \%$ in published case series [24]. Other important underlying diagnoses include acute liver failure, autoimmune liver disease, alpha-1-antitrypsin deficiency, metabolic liver disease and hepatoblastoma, all with shares between 5 and $8 \%$ [24], underlining the heterogeneity of the pediatric transplant population. It is easily conceivable that primary diagnoses as different as hepatoblastoma (with or without chemotherapy), autoimmune liver disease and biliary atresia might lead to very different immune layouts before transplantation, with uncertain impact on the reaction to immunosuppression after transplantation. In addition, age has been shown to have a major impact on circulating $\mathrm{TH} 1$ and $\mathrm{TH} 2$ cytokine levels that is irrespective of immunosuppressive therapy or rejection [9]. These findings underline why results from adult studies cannot be easily transferred to children. Any study on immune monitoring in children will have to take into account differences and characteristics that might result from age and development.

To our knowledge, all published pediatric studies are single-center studies with small patient numbers. Our study will benefit from a multicenter approach in order to obtain an increase in sample size that will permit differentiated analysis.

Our study proposes a longitudinal rather than a crosssectional approach. In previous studies that focused on longitudinal evaluations of immune markers after solid organ transplantation the main differences that could be found between rejectors and non-rejectors were intraindividual changes in immune markers when compared to baseline. A study on 40 children with intensive follow-up until day 28 after transplantation described distinct trajectories of circulating cytokine levels [8]. Differences in early trajectories were associated with the occurrence of early BPAR. In another study on adult transplant recipients, a spike in TH1 cytokines around rejection episodes was observed [11]. Our longitudinal approach will allow for a better understanding of the early changes after transplantation anticipating the baseline state of innate and adaptive immune system before transplantation. Our evaluation will clarify whether there are specific reaction patterns in the immune system that can be recognized at an early time point and that have a predictive meaning for the further immune response of the patient towards the occurrence of rejection or infections at a later date. The analysis in our proposed study will incorporate rejections throughout the whole first year after transplantation. Rather than performing a step by step comparison or comparison to baseline, our statistical approach will focus on taking into account the entire trajectory of the different patterns of immune reaction from pre transplantation throughout the first year after transplantation.

Immune markers tested in previously published studies used various methods for assessing $\mathrm{T}$ cell activation and proliferation, immune cell numbers and circulating cytokines. As discussed above, an ideal monitoring tool would be easy to use in routine clinical care, yield rapid results that enable dose adjustments and a timely control of their effect and would be cost-effective. We have chosen immune cell numbers by Trucount analysis and circulating cytokines and chemokines as potential immune markers.

The host's immune response against the graft is based on a combination of different immune cells [25]. The recognition of allo-antigens is based on an interaction of foreign donor major histocompatibility complex (MHC) $\mathrm{I}$ and II molecules with recipient CD8+ and $\mathrm{CD} 4+\mathrm{T}$ cells. B cells, dendritic cells and natural killer (NK) cells all exist in inflammatory and anti-inflammatory subpopulations and are hence likely to contribute to allograft rejection [25]. Trucount analysis will facilitate monitoring the balance between pro- and anti-inflammatory subpopulations and uncover expansion of effector populations. Previous studies have shown that rather than absolute cell numbers it is the balance between pro- and anti-inflammatory immune phenotypes that determines outcome [5, 9]. In addition, the Trucount technology offers comparatively quick results. In view of the quantity of samples to be analyzed and the future applicability for clinical practice it appears necessary to restrict the analysis to quantification of different immune cells, but omit the technically more advanced and complicated analysis of e.g. $\mathrm{T}$ cell proliferation or activation used in other studies.

Soluble cytokine levels in the peripheral blood represent a compound measure of activation of different immune cells. One disadvantage of this technique is that peri-surgical stress, infection and ischemia-reperfusion injury might also contribute to circulating cytokines and therefore, represent confounding factors at the immediate early phase after transplantation, i.e. within 24 to $48 \mathrm{~h} \mathrm{[8].} \mathrm{However,} \mathrm{previous} \mathrm{studies} \mathrm{have} \mathrm{clearly} \mathrm{linked}$ individual peripheral cytokines to immune status and occurrence of rejection after transplantation $[8-10,26]$. Thus, analysis in peripheral blood offers the advantage of easily obtainable material and with even small sample 
volumes of less than $2 \mathrm{ml}$, which is suitable for children, clinically relevant information can be obtained and provide an insight into the recipient's immune status. Our proposed multiplex analysis allows the determination of blood levels of up to 50 different cytokines and chemokines in a minimal amount of plasma. This combines the advantage of a thorough and differentiated evaluation of immune markers incorporating both pro- and anti-inflammatory markers with adequate feasibility in a pediatric setting where available quantities of blood are often very small.

\section{Summary}

In summary, we propose a prospective longitudinal multicenter cohort study on immune monitoring after pediatric liver transplantation. This study is designed to enhance and expand existing knowledge on the immune response to immunosuppression after liver transplantation in children. Identification of trajectories of immune markers over time will facilitate the understanding of different types of immune reaction that might lead to BPAR. Moreover, the study will help to understand whether these different immune reactions are consequences of primary disease, developmental differences, or of innate differences within the individual immune system. The overall aim of our study is the detection of immune markers that will enable us to guide immunosuppressive therapy in the future.

\section{Abbreviations}

AUC: Area under the curve; BPAR: Biopsy-proven acute rejection; CSA: Cyclosporin A; eCRF: Electronic case report form; IS: Immunosuppression; MHC: Major histocompatibility complex; MMF: Mycophenolic acid; NK: Natural killer; Tx: Transplantation

\section{Funding}

Public funding sources:

- Grant by the German Federal Ministry of Education and Research (reference number 01EO1302): partial funding of clinician scientist at Hannover Medical School and of laboratory analyses. No involvement in study design, analysis or interpretation of data or in writing the manuscript.

- Network grant by the ESPGHAN (European Society of Paediatric Gastroenterology, Hepatology and Nutrition). Funding of generation and implementation of the electronic case report form. No involvement in study design, analysis or interpretation of data or in writing the manuscript.

Industrial funding sources

- Astellas pharma: funding of sample transport and data collection at collaborating centres. No involvement in study design, analysis or interpretation of data or in writing the manuscript.

Private funding sources

- Appenroth foundation: funding of data documentation at Hannover Medical School. No involvement in study design, analysis or interpretation of data or in writing the manuscript.

\section{Authors' contributions}

UB, IG, CF, AK and RM developed the study design and initiated the study. UB and IG initiated the international cooperation and facilitated development and implementation of the eCRF. IG, FM, EP and NJ are in charge of patient enrolment and data acquisition at Hannover Medical School. IG and UB provide analysis and interpretation of clinical data. Ld'A, $\mathrm{PM}, \mathrm{DK}, \mathrm{DD}, \mathrm{VM}, \mathrm{JP}$ and $\mathrm{LH}$ are principal investigators at the international cooperating centres. They have made important contributions towards study design and study layout and and are in charge of local data acquisition and documentation. AK, TM and RM provide the statistical concepts for the study and made important contributions towards study design. CF, KD and JK provide analysis, documentation and interpretation of immunological data. All authors have read and approved the final manuscript.

\section{Ethics approval and consent to participate}

Primary approval was granted by the ethics committee of Hannover Medical School (Chair: Prof. Dr. H.D. Tröger) under the reference umber 1596-2012. The study protocol was then approved by the local ethics committees of all participating centers (Research Ethics Committee, Birmingham Children's Hospital NHS Foundation Trust, Birmingham (UK); Ethics Committee of the "Papa Giovanni XXIII", Bergamo (Italy); Ethics Committee of the Children and Adolescents' Department of the University Hospital Geneva, Geneva (Switzerland); Ethics Committee of Clinical Research of the University Hospital La Paz, Madrid (Spain); Committee for the Protection of Persons lle de France II (CPP IDF II), Paris (France); Bioethics Commission of the Institute PomnikCentrum Zdrowia Dziecka (IPCZD, Centre of Child Health), Warszawa (Poland)). Informed written consent for study participation was obtained by parents or legal guardians of all study participants, and by study participants themselves if aged $>16$ yrs.

\section{Competing interests}

The authors declare that they have no competing interests.

\section{Publisher's Note}

Springer Nature remains neutral with regard to jurisdictional claims in published maps and institutional affiliations.

\section{Author details}

'Division of Paediatric Gastroenterology and Hepatology, Department of Paediatric Liver, Kidney and Metabolic Diseases, Hannover Medical School, Carl-Neuberg-Strasse 1, 30625 Hannover, Germany. ${ }^{2}$ Epidemiological and Statistical Methods Research Group, Helmholtz Centre for Infection Research, Inhoffenstr. 7, 38127 Braunschweig, Germany. ${ }^{3}$ Paediatric Liver, Gl and Transplantation, Ospedali Riuniti di Bergamo, Largo Barozzi 1, 24128 Bergamo, Italy. ${ }^{4}$ Liver Unit, Birmingham Childrens Hospital, Steelhouse Lane, Birmingham B4 6NH, UK. ${ }^{5}$ Pédiatre Hépatologue, Service d'Hépatologie-Gastroentérologie-Nutrition, Hôpital Necker-Enfants Malades, 149 rue de Sèvres, 75015 Paris, France. ${ }^{6}$ Hopitaux Universitaires de Geneve, Hopital des Enfants pt Pédiatrie, Serv. Spécialités Pédiatriques, Rue Gabrielle-Perret-Gentil 4, 1211 Genève 4, Switzerland. ${ }^{7}$ Centrum Zdrowia Dziecka, Al. Dzieci Polskich 20, 04-730 Warszawa, Poland. ${ }^{8}$ Servicio de Hepatologia y Transplante, Hospital Infantil Universitario La Paz Madrid, Paseo de la Castellana 261, 28046 Madrid, Spain. ${ }^{9}$ Institute of Transplant Immunology, IFB-Tx, Hannover Medical School, Car-Neuberg-Str. 1, 30625 Hannover, Germany. ${ }^{10}$ Institute for Medical Epidemiology, Biometrics and Informatics, Martin-Luther-University Halle-Wittenberg, 06097 Halle (Saale), Germany. ${ }^{11}$ Paediatric Hepatology Program, Children's Hospital of Pittsburgh, One Children's Hospital Way, 4401 Penn Ave, Pittsburgh, PA 15224, USA.

Received: 2 November 2017 Accepted: 8 May 2018

Published online: 16 May 2018

\section{References}

1. Sawitzki B, Reinke P, Pascher A, Volk HD. State of the art on the research for biomarkers allowing individual, tailor-made minimization of immunosuppression. Curr Opin Organ Transplant. 2010;15(6):691-6.

2. Levitsky J. Next level of immunosuppression: drug/immune monitoring. Liver Transpl. 2011;17(Suppl 3):S60-5.

3. Millan O, Rafael-Valdivia L, Torrademe E, Lopez A, Fortuna V, Sanchez-Cabus $\mathrm{S}$, et al. Intracellular IFN-gamma and IL-2 expression monitoring as surrogate 
markers of the risk of acute rejection and personal drug response in de novo liver transplant recipients. Cytokine. 2013;61(2):556-64.

4. Millan O, Rafael-Valdivia L, San SD, Boix F, Castro-Panete MJ, Lopez-Hoyos M, et al. Should IFN-gamma, IL-17 and IL-2 be considered predictive biomarkers of acute rejection in liver and kidney transplant? Results of a multicentric study. Clin Immunol. 2014;154(2):141-54.

5. Stalder M, Birsan T, Holm B, Haririfar M, Scandling J, Morris RE. Quantification of immunosuppression by flow cytometry in stable renal transplant recipients. Ther Drug Monit. 2003;25(1):22-7.

6. Barten MJ, Rahmel A, Bocsi J, Boldt A, Garbade J, Dhein S, et al. Cytokine analysis to predict immunosuppression. Cytometry A. 2006;69(3):155-7.

7. Giese T, Zeier M, Schemmer P, Uhl W, Schoels M, Dengler T, et al. Monitoring of NFAT-regulated gene expression in the peripheral blood of allograft recipients: a novel perspective toward individually optimized drug doses of cyclosporine a. Transplantation. 2004;77(3):339-44.

8. Gras J, Wieers G, Vaerman JL, Truong DQ, Sokal E, Otte JB, et al. Early immunological monitoring after pediatric liver transplantation: cytokine immune deviation and graft acceptance in 40 recipients. Liver Transpl. 2007; 13(3):426-33.

9. Ganschow R, Broering DC, Nolkemper D, Albani J, Kemper MJ, Rogiers X, et al. Th2 cytokine profile in infants predisposes to improved graft acceptance after liver transplantation. Transplantation. 2001;72(5):929-34.

10. Conti F, Frappier J, Dharancy S, Chereau C, Houssin D, Weill B, et al. Interleukin-15 production during liver allograft rejection in humans. Transplantation. 2003;76(1):210-6.

11. Minguela A, Torio A, Marin L, Sanchez-Bueno F, Garcia-Alonso AM, Ontanon J, et al. Implication of Th1, Th2, and Th3 cytokines in liver graft acceptance. Transplant Proc. 1999;31(1-2):519-20.

12. Truong DQ, Cornet A, Wieers G, Robert A, Reding R, Latinne D. Preand post-transplant monitoring of granzyme B enzyme-linked immunosorbent spot assay in pediatric liver recipients. Transpl Immunol. 2008;19(3-4):215-9.

13. Kim Y, Park KH, Chung BH, Choi BS, Yang CW, Kim Jl, et al. Pretransplant IFN-gamma ELISPOT assay as a potential screening test to select immunosuppression protocols for patients receiving basiliximab induction therapy. Transl Res. 2012;160(3):230-6.

14. Rizopoulos D, Ghosh P. A Bayesian semiparametric multivariate joint model for multiple longitudinal outcomes and a time-to-event. Stat Med. 2011; 30(12):1366-80.

15. Waldmann E, Taylor-Robinson D, Klein N, Kneib T, Pressler T, Schmid M, et al. Boosting joint models for longitudinal and time-to-event data. Biom J. 2017;59(6):1104-21.

16. Proust-Lima C, Dartigues JF, Jacamin-Gadda H. Joint modeling of repeated multivariate cognitive measures and competing risks of dementia and death: a latent process and latent class approach. Stat Med. 2016;35(3):382-98.

17. Rizopoulos D. Dynamic predictions and prospective accuracy in joint models for longitudinal and time-to-event data. Biometrics. 2011;67(3):81929.

18. Perrella O, Sbreglia C, Arenga G, Perrella A, Ferrara A, D'Antonio A, et al. Acute rejection after liver transplantation: is there a specific immunological pattern? Transplant Proc. 2006;38(10):3594-6.

19. Masri MA, Stephan A, Barbari S, Rizk A, Kamel G. Pre- and posttransplant immunologic monitoring: why, when, and how? Transplant Proc. 2002;34(6): 2482-4.

20. Kim SH, Oh EJ, Kim MJ, Park YJ, Han K, Yang HJ, et al. Pretransplant donor-specific interferon-gamma ELISPOT assay predicts acute rejection episodes in renal transplant recipients. Transplant Proc. 2007;39(10): 3057-60.

21. Rodrigo E, Lopez-Hoyos M, Corral M, Fabrega E, Fernandez-Fresnedo G, San SD, et al. Immuknow as a diagnostic tool for predicting infection and acute rejection in adult liver transplant recipients: a systematic review and metaanalysis. Liver Transpl. 2012;18(10):1245-53.

22. Millan O, Brunet M. Cytokine-based immune monitoring. Clin Biochem. 2016;49(4-5):338-46.

23. Brunet M, Millan LO, Lopez-Hoyos M. T-cell cytokines as predictive markers of the risk of allograft rejection. Ther Drug Monit. 2016;38(Suppl 1):S21-8.

24. Goldschmidt I, Stieghorst H, Munteanu M, Poynard T, Schlue J, Streckenbach $C$, et al. The use of transient elastography and non-invasive serum markers of fibrosis in pediatric liver transplant recipients. Pediatr Transplant. 2013; 17(6):525-34.
25. Sawitzki B, Schlickeiser S, Reinke P, Volk HD. Monitoring tolerance and rejection in organ transplant recipients. Biomarkers. 2011;16(Suppl 1):S42-50.

26. Millan O, Benitez C, Guillen D, Lopez A, Rimola A, Sanchez-Fueyo A, et al. Biomarkers of immunoregulatory status in stable liver transplant recipients undergoing weaning of immunosuppressive therapy. Clin Immunol. 2010; 137(3):337-46.

\section{Ready to submit your research? Choose BMC and benefit from:}

- fast, convenient online submission

- thorough peer review by experienced researchers in your field

- rapid publication on acceptance

- support for research data, including large and complex data types

- gold Open Access which fosters wider collaboration and increased citations

- maximum visibility for your research: over $100 \mathrm{M}$ website views per year

At BMC, research is always in progress.

Learn more biomedcentral.com/submissions 